\title{
Adipose-derived stem cell therapy for severe muscle tears in working German shepherds: Two case reports
}

\author{
S. Gary Brown ${ }^{1}$, Robert J. Harman ${ }^{2}$, Linda L. Black ${ }^{2}$ \\ ${ }^{1}$ Veterinary Orthopedic and Surgery Service, Western University of Health Sciences, Pomona, USA; gary@vetortho.com \\ ${ }^{2}$ Vet-Stem Inc., Poway, USA; LBlack@Vet-Stem.com
}

Received 21 January 2012; revised 24 February 2012; accepted 31 March 2012

\begin{abstract}
Injuries to muscle in the elite athlete are common and may be responsible for prolonged periods of loss of competitive activity. The implications for the athlete, his/her coach and team may be catastrophic if the injury occurs at a critical time in the athlete's diary. Imaging now plays a crucial role in diagnosis, prognostication and management of athletes with muscle injuries. This article discusses the methods available to clinicians and radiologists that are used to assess skeletal muscle injury. The spectrum of muscle injuries sustained in the elite athlete population is both discussed and illustrated.
\end{abstract}

Keywords: Adipose-Derived; Stem Cell; Dog; Muscle

\section{INTRODUCTION}

Muscle injuries occur frequently in athletic dogs and athletes [1]. Traditional therapies, such as non-steroidal anti-inflammatory administration, rest, antioxidant therapy and physical therapy have generally not been helpful in preventing fibrosis and permanent muscle contracture, which leads to functional impairment. Similar findings occur in human medicine in that similar suggested treatments for muscle injury are generally not sufficient to enhance muscle regeneration and prevent fibrosis [2].

Depending on their severity, muscle strains may be categorized into three grades [1]: Grade 1 is a mild strain with damage to individual muscle fibers with no hematoma formation; Grade 2 is a more extensive tear, with increased muscle fiber involvement, with no complete rupture [3], localized swelling and heat and slight lameness; and Grade 3 is a complete rupture of a muscle with major disruption of the muscle fibers and hematoma formation, palpable disruption, and significant pain [1].

Inflammation, the hallmark of acutely injured muscle [4], initiates a rapid invasion of the muscle by inflammatory cell populations that can persist for days to weeks. The inflammation phase is followed, and partially coexists with the muscle repair, regeneration, and growth phases. Neutrophils migrate to injured muscle followed by macrophages that remove damaged myofibers [5] and secrete factors that are chemotactic for muscle precursor cells [6]. Macrophages have also been implicated in muscle damage via the release of nitric oxide and through promotion of membrane damage in non-diseased muscle [4]. Although new muscle fibers regenerate after injury, the healing progress is very slow and can lead to fibrosis and functional impairment [6].

Biological approaches to enhance muscle regeneration and prevent fibrosis are being investigated in animal models and human medicine [6]. Because of the lack of therapeutic options that decrease fibrosis, significant efforts are being made to improve the current treatment of skeletal muscle injury using a regenerative medicine approach [2]. Matziolus et al. demonstrated that autologous bone marrow-derived cells enhance muscle strength following skeletal muscle crush injury in rats [7]. The same group later reported that rats with severe skeletal muscle injury treated with mesenchymal stem cells responded in a dose dependent manner with an increasing logarithmic response in muscle contractile force as the dose of MSCs was increased [8]. Muscle derived stem cells used in skeletal muscle injury in mice promote angiogenesis and decrease fibrosis [9]. Merritt and colleagues used MSCseeded extracellular matrix (ECM) to treat skeletal muscle injury in rats. This group demonstrated that the implanted MSC-seeded ECM had more blood vessels and regenerating skeletal myofibers than the ECM without cells $(p<0.05)$. There is ample literature to support the use of adult stem cells in the treatment of muscle injury.

Adipose-derived stem cells (ADSC) have been the fo- 
cus of recent reviews [10] and studies [9,11-13]. Autologous ADSCs have been used in veterinary medicine since 2003. However, there are no reports to our knowledge of treatment with ADSCs or other stem cells, in dogs with muscle injury. Two working, male German shepherd dogs presented for acute semitendinosus muscle injuries in one or both hind limbs. The surgeon confirmed the clinical diagnosis of muscle tear using ultrasound. At that time, approximately 60 grams of fat was harvested from the falciform ligament in each, and the ADSC (stromal vascular fraction) extracted by Vet-Stem, Inc. (Poway, CA) [11]. Forty-eight hours later, the dogs were placed under anesthesia and the ADSC aliquot was injected with a 22 gauge needle by digital palpation directly into the muscle, both intralesional and paralesional, and intravenously. Follow-up ultrasound evaluations were performed six to ten weeks after stem cell therapy. Muscle biopsy was not performed because of potential increased morbidity in a clinical case. New needle biopsy techniques might be considered in the future. MRI was not available in this practice. Outcome measurements for the case reports included owner assessments (visual assessment of dog's improvement in affected tasks) and surgeon assessments (flexibility and agility from normal to very severe) before and at end of treatment period, and the ability of the dog to return to work.

\section{CASE REPORTS}

Cris, a four-year-old male German shepherd police dog, became suddenly lame while training. Five days later, the owner reported that the dog's lameness had improved but that the dog's gait remained abnormal. Cris presented at that time bearing less weight on the left rear limb with a slight circumduction gait. On palpation, the distal two-thirds of the left semitendinosus muscle was

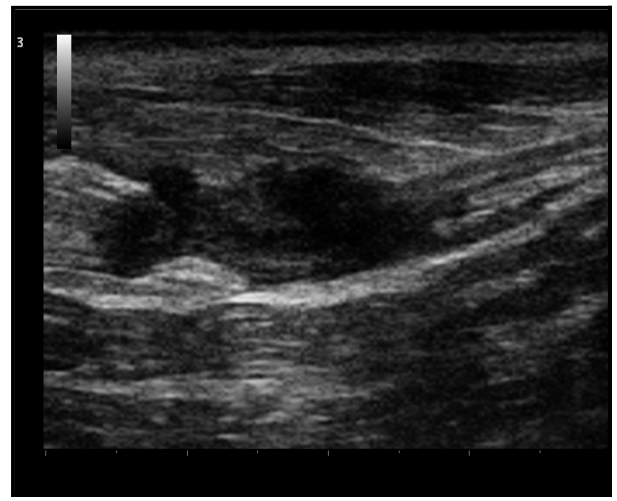

(a) swollen at the mid belly region with a palpable dimple. Sonography revealed poorly defined hypoechoic region in the distal two-thirds of the left semitendinosus muscle $8.1 \mathrm{~mm}$ wide $\times 30.8 \mathrm{~mm}$ long. These findings are compatible with a focal tear in the left semitendinosus muscle with an area of central hemorrhage and/or edema consistent with a grade 3 tear (Figure 1). No abnormalities were seen in the right semitendinosus muscle. At that time, the surgeon prescribed Previcox (227 mg 5 days/ week) and antioxidant therapy (Niacinamide, $50 \mathrm{mg} /$ day; Sam-E, 225 mg/day; Vitamin E, 200 IU/day; 3V Caps, 1 cap/day; and Omega Mint, 1/day) and the dog was prescribed cage rest for two weeks.

After two weeks, Cris had not responded to traditional therapy and the owner elected to treat with stem cells. After fat collection and stem cell isolation as previously described [11], the surgeon injected approximately 4.7 million ADSCs into the muscle injury site. Approximately 4.7 million cells were injected intravenously (IV). Antioxidant therapy was continued. Cris was walked each day for one month then began light training. Sonographic examination at 82 days post injection revealed significant reduction in lesion size, $11 \mathrm{~mm}$ wide $\times 15 \mathrm{~mm}$ long (Figure 2(a)-pre, (b)-post) and an increased number of fibers coursing through this area that appeared organized and oriented in the same plane as the normal muscle. Clinically the dog was normal with no lameness or gait abnormalities. On the owner evaluation, Cris improved from moderately affected before therapy to normal within 19 weeks after therapy. The surgeon's evaluation of Cris revealed him to have severely affected flexibility and moderately affected agility before therapy that improved to normal 19 weeks after the stem cell injection. Telephone conversation 21 months post injection reveals that he was in service and completely normal.

Jago, an eight-year-old male German shepherd police

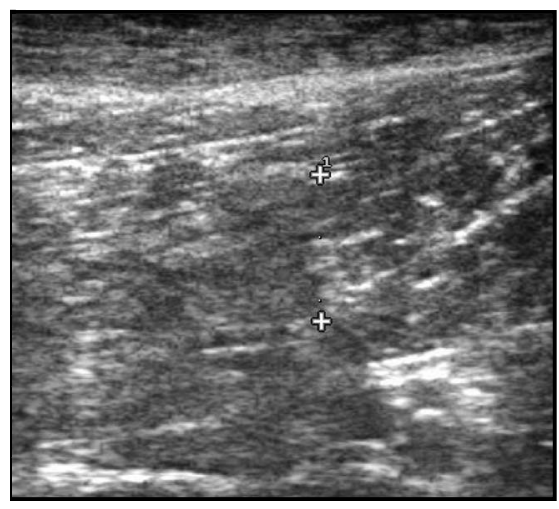

(b)

Figure 1. (a) Ultrasound scan of Cris left ST muscle tear before treatment: An $8 \mathrm{~mm}$ wide and $30 \mathrm{~mm}$ long poorly defined hypoechoic area is noted in the distal two thirds of the ST muscle compatible with a muscle tear; (b) Cris 82 days after treatment: The lesion is poorly defined with an iso-echoic area that measured $11 \mathrm{~mm}$ wide and $15 \mathrm{~mm}$ in length. An increase in fiber numbers is noted that are oriented in the same plane as the normal muscle. 


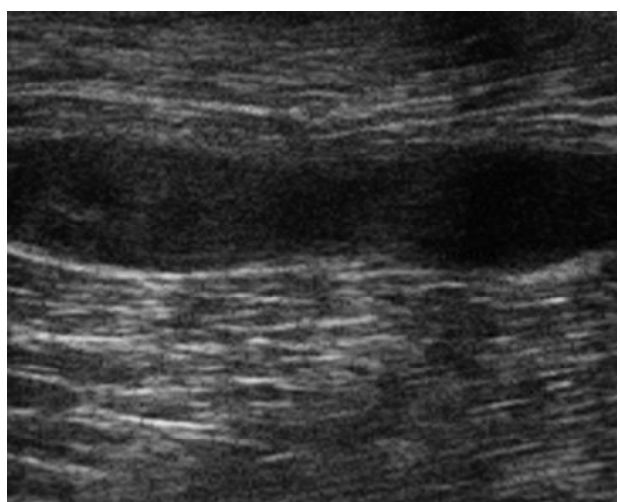

(a)

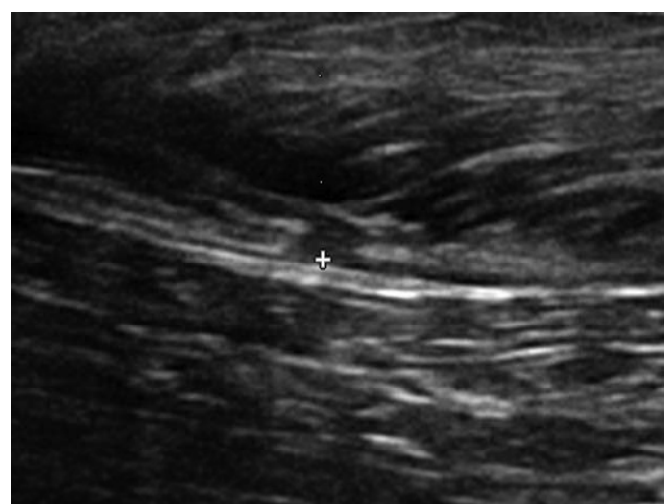

(b)

Figure 2. (a) Jago sonogram before treatment. A $9 \mathrm{~mm}$ wide $\times 6 \mathrm{~cm}$ long hypoechoic area exists in the cranial aspect of the right semitendinosus muscle consistent with a muscle tear. Generalized disruption of the muscle fiber pattern with no organized hyperechoic areas suggestive of scarring; (b) 11 weeks after ADSC treatment. The previously seen hypoechoic area decreased in size to measure $5 \mathrm{~mm}$ wide $\times 2.4 \mathrm{~cm}$ in length. An increase in fiber pattern development with fibers running parallel to the orientation of the muscle was observed.

dog, was injured during a suspect apprehension and presented seven days after injury with moderate to severe lameness and semitendinosus tenderness and swelling. Both rear limbs were affected. He was diagnosed clinically and sonographically with a grade 2.5 acute on chronic injury to his right semitendinosus muscle as demonstrated by ultrasound (Figure 2(a)-pre) and a grade 1 acute on chronic injury to his left semitendinosus muscle (not shown). The right muscle was about 30 percent enlarged on palpation and the dog had a circumduction gait in the right rear limb. The left muscle had no palpable enlargement, was not tender to palpation, and he had no gait abnormality, but had a history of stiffness. Jago was treated with 7.5 million ADSC in each muscle lesion and administered 3.8 million cells IV. He underwent physical therapy and antioxidant therapy, and was prescribed deracoxib (37.5 mg SID). Jago underwent one month of a progressively increased walking regimen prior to resuming light training at 105 days.

Twenty-two (22) weeks after injection Jago was markedly improved. His surgeon scores for agility and flexibility improved from severely affected to mildly affected. His owner evaluations were markedly improved. Jago competed in a two-day police dog competition and placed second in protection and third place in obedience 22 weeks post ADSC injection.

\section{DISCUSSION}

The beneficial effects of ADSC therapy in muscle injury are likely explained by the local secretion of cytokines and growth factors [14-16]. Natsu et al. treated skeletal muscle laceration with bone marrow-derived MSCs, and the muscle improved functionally without evidence of fusion or differentiation of the injected cells suggesting that mechanisms other than differentiation were responsible for the benefit seen [16]. ADSCs are also immunomodulatory, which may play a therapeutic role $[17,18]$.

\section{CONCLUSION}

ADSC therapy enhanced muscle healing and prevented fibrosis in these clinical cases, similar to the reported literature in laboratory animals. The dogs returned to their previous training and occupations with a functional gait and no lameness. This is the first report of using stem cells in dogs to treat skeletal muscle injury. Further controlled clinical trials are necessary for the complete evaluation of the therapeutic benefit of ADSC therapy in canine muscle injuries.

\section{ACKNOWLEDGEMENTS}

The authors wish to thank Dr. David Detweiller, ACVR, for performing the sonograms and for his evaluations and comments. We thank Karen Lavrischeff, RVT for her editorial assistance.

\section{REFERENCES}

[1] Lee, J.C., et al. (2012) Imaging of muscle injuries. British Journal of Radiology, 2012. doi:10.1259/bjr/84622172

[2] Quintero, A., Wright, V., Fu, F., et al. (2009) Stem cells for the treatment of skeletal muscle injury. Clinics in Sports Medicine, 28, 1-11. doi:10.1016/j.csm.2008.08.009

[3] Lewis, D., Shelton, D., Piras, A., et al. (1997) Gracilis or semitendinosus myopathy in 18 dogs. Journal of the American Animal Hospital Association, 33, 177-188.

[4] Tidball, J. (2005) Inflammatory processes in muscle injury and repair. American Physiological Society American Journal of Physiology-Regulatory, Integrative and Com- 
parative Physiology, 288, 345-353.

[5] Hannafin, J.A., Pedowitz, R.A., Hidaka, C., et al. (1994) Pathophysiology and healing of musculoskeletal tissues. In: Griffin, L.Y., Ed., Orthopaedic Knowledge Update: Sports Medicine, American Academy of Orthopaedic, Rosemont, 17-33.

[6] Fukushima, K., Badlani, N., Usas, A., et al. (2001) The use of an antifibrosis agent to improve muscle recovery after laceration. The American Journal of Sports Medicine, 29, 394-402.

[7] Matziolis, G., Winkler, T., Schaser, K., Wiemann, M., et al. (2006) Autologous bone marrow-derived cells enhance muscle strength following skeletal muscle crush injury in rats. Tissue Engineering, 12, 361-367. doi:10.1089/ten.2006.12.361

[8] Winkler, T., von Roth, P., Matziolis, G., et al. (2009) Doseresponse relationship of mesenchymal stem cell transplantation and functional regeneration after severe skeletal muscle injury in rats. Tissue Engineering, 15, 487-494. doi:10.1089/ten.tea.2007.0426

[9] Ota, S., Uehara, K., Nozaki, M., et al. (2011) Intramuscular transplantation of muscle-derived stem cells accelerates skeletal muscle healing after contusion injury via enhancement of angiogenesis. The American Journal of Sports Medicine, 9, 1912-1922. doi:10.1177/0363546511415239

[10] Gimble, J., Bunnell, B., Chiu, E., et al. (2011) Concise Review: Adipose-derived stromal vascular fraction cells and stem cells: Let's not get lost in translation. Stem Cells, 29, 749-754. doi:10.1002/stem.629

[11] Black, L., Gaynor, J., Gahring, D., et al. (2007) Effect of adipose-derived mesenchymal stem and regenerative cells on lameness in dogs with chronic osteoarthritis of the coxofemoral joints: A randomized, double-blinded, multicenter, controlled trial. Veterinary Therapeutics, 8, 272 284.

[12] Dahlgren, L.A. (2006) Use of adipose derived stem cells in tendon and ligament injuries. Proceedings of American College of Veterinary Surgeons-Veterinary Symposium Equine and Small Animal, 35, E5.

[13] Nixon, A., Dahlgren, L., Haupt, J., et al. (2008) Effect of adipose-derived nucleated cell fractions on tendon repair in a collagenase-induced tendinitis model. American Journal of Veterinary Research, 69, 1-10. doi:10.2460/ajvr.69.7.928

[14] Caplan, A. and Diego, C. (2011) The MSC: An injury drugstore. Cell Stem Cell, 9, 11-15. doi:10.1016/j.stem.2011.06.008

[15] Kilroy, G., Foster, S., Wu, X., et al. (2007) Cytokine profile of human adipose-derived stem cells: Expression of angiogenic, hematopoietic, and pro-inflammatory factors. Journal of Cellular Physiology, 212, 702-709. doi:10.1002/jcp.21068

[16] Natsu, K., et al. (2004) Allogeneic bone marrow-derived mesenchymal stromal cells promote the regeneration of injured skeletal muscle without differentiation into myofibers. Tissue Engineering, 10, 1093-1112. doi:10.1089/ten.2004.10.1093

[17] Le Blanc, K. (2006) Mesenchymal stromal cells: Tissue repair and immune modulation. Cytotherapy, 8, 559-561.

[18] McIntosh, K., Zvonic, S., Garrett, S., et al. (2006) The immunogenicity of adipose derived cells: Temporal changes in vitro. Stem Cells, 24, 1246-1253. doi:10.1634/stemcells.2005-0235 